\title{
APLICACIONES ELECTRONICAS DEL AUTOMOVIL 2
}

\section{AUTOMOBILE ELECTRONIC APPLICATIONS 2}

\section{AUTORES}

Arturo Pérez París: Alumno de la Escuela Politécnica de la Universidad de Alcalá arturo.perez@aol.com

\section{CURRÍCULUM VITAE}

Alumno de la Escuela Politécnica de la Universidad de Alcalá de Henares (España). Ingeniero de Soporte Técnico en Kone Elevadores

\section{RESUMEN}

Conviene, antes de comenzar con la descripción de sistemas electrónicos de encendido de motores de explosión, repasar el principio de funcionamiento de un sistema convencional de encendido y analizar cuáles son los problemas que este tipo de mecanismos presenta, para posteriormente explicar las soluciones que aportan los sistemas electrónicos.

\section{PALABRAS CLAVE}

Automóvil - Mecánica - Motores

\section{ABSTRACT}

It should, before beginning the description of electronic ignition internal combustion engines, review the working principle of a conventional system on and analyze what 
are the problems that this type of mechanism presents, and later explain the solutions provided by the electronic systems.

\section{KEY WORDS}

Automotive - Mechanical - Motors

En los anteriores artículos sobre las aplicaciones de la electricidad y la electrónica en el automóvil se trataron los sistemas auxiliares. En este estudiaremos un elemento fundamental :

El circuito de encendido del motor

Conviene, antes de comenzar con la descripción de sistemas electrónicos de encendido de motores de explosión, repasar el principio de funcionamiento de un sistema convencional de encendido y analizar cuáles son los problemas que este tipo de mecanismos presenta, para posteriormente explicar las soluciones que aportan los sistemas electrónicos. 
Un sistema de ignición convencional como:

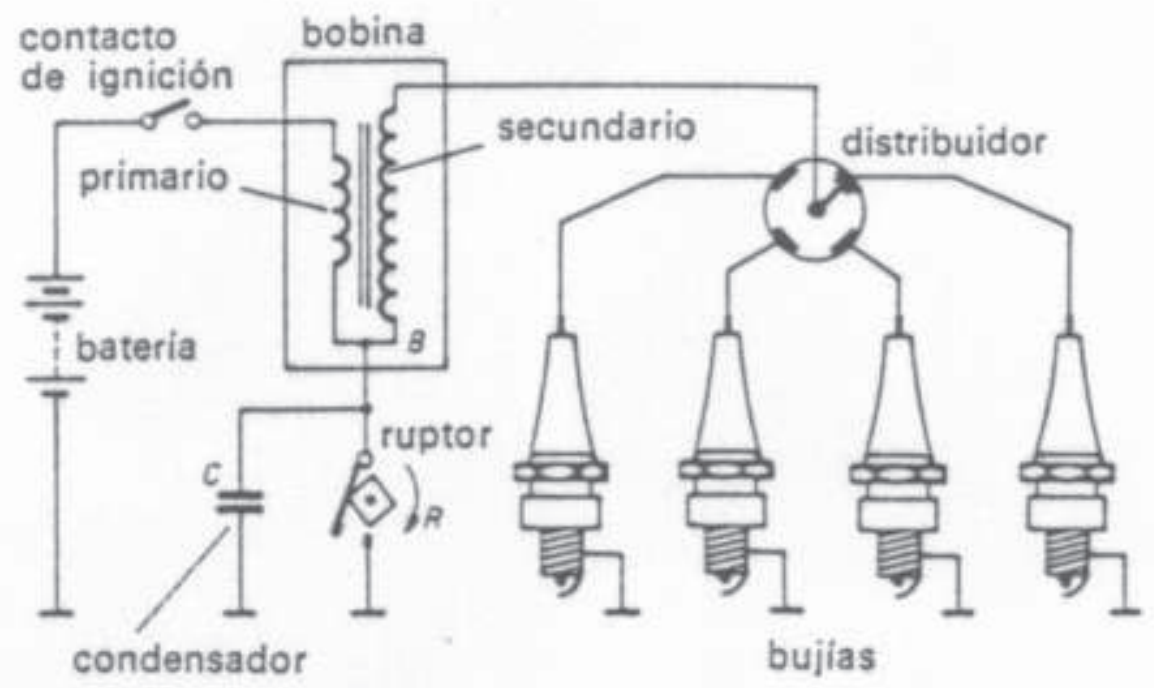

utiliza, para producir la chispa en las bujías, la energía almacenada en el primario de una bobina durante el tiempo en el que un ruptor (platinos) está cerrado, energía que se libera al abrirse éste. El condensador en paralelo con el ruptor hace de moderador, haciendo que la descarga de la energía de la bobina no sea excesivamente rápida; de manera que dicha energía se pueda transferir al secundario de la bobina y la chispa salte en las bujías y no en el ruptor. Completa el sistema un distribuidor para conectar al circuito la bujía que corresponda.

El proceso que se desarrolla en el circuito para la producción de la chispa y las magnitudes que ahí se manejan son las siguientes: En el momento en que se cierra el ruptor, la corriente que atraviesa el primario de la bobina crece hasta que al cabo de un tiempo, alcanza el 95\% del valor que le permite su resistencia óhmica. En estas circunstancias la bobina alcanza una energía:

$\mathrm{W}=(\mathrm{Lp} \times \mathrm{I} 2) / 2$ 
siendo: $\mathrm{Lp}=$ inductancia del primario, e I = intensidad del primario que es igual a 0,95 E / Rp, donde E es la tensión de alimentación y Rp la resistencia óhmica del primario.

El tiempo necesario para alcanzar el 95\% de la corriente viene dado por la expresión:

$$
t=3 \cdot \frac{L_{y}}{R_{y}}
$$

Considerando que los valores de las bobinas comerciales suelen estar alrededor de :

Inductancia del primario: $\mathrm{Lp}=12 \mathrm{mH}$.

Inductancia del secundario: $\mathrm{Ls}=66 \mathrm{H}$.

Resistencia del primario: $\mathrm{Rp}=3,5 \mathrm{~W}$.

Resistencia del secundario: $\mathrm{Rs}=9 \mathrm{~kW}$.

Relación de transformación real: $\mathrm{n}=68$.

Resulta que para una tensión de alimentación de $12 \mathrm{~V}$, se obtiene una corriente de :

$$
I=0,95 \cdot \frac{E}{R_{p}}=0,95 \times \frac{12}{3,5}=3,26 \mathrm{~A}
$$

una energía de:

$$
W=\frac{1}{2} \cdot L_{y} \cdot I^{2}=\frac{1}{2} \times 12 \times 3,26^{2}=63,7 \mathrm{~mJ}
$$

y un tiempo de:

$$
t=3 \cdot \frac{L_{y}}{R_{y}}=3 \times \frac{12}{3,5}=10,3 \mathrm{~ms}
$$


Lo cual significa que, en un motor de cuatro tiempos y cuatro cilindros, en el que por cada revolución del motor se producen dos chispas de encendido a diferentes cilindros, el número de revoluciones máximo que permite obtener la energía de chispa calculada (correspondiente al 95\% de la máxima obtenible) no debe ser superior en números redondos a 3.000 revoluciones por minuto.

Esta situación conlleva el que, a mayor número de revoluciones, la intensidad a través del primario sólo alcanza una parte de la que permite la bobina, decreciendo consecuentemente la energía de la chispa. Hay que tener en cuenta también que, en el cálculo anterior, no se ha considerado el tiempo que permanece abierto el ruptor, que es durante el que se produce la chispa y que, a velocidades de rotación elevadas, el ruptor, debido a la inercia propia de un sistema mecánico, tiende a vibrar y, por tanto, a estar cerrado menos tiempo.

Del análisis anterior es fácil de deducir los inconvenientes que presenta el sistema convencional:

El ruptor es un elemento mecánico y, como tal, tiene los problemas inherentes a los sistemas mecánicos: inercias, vibraciones, etc.

Por el ruptor pasan corrientes relativamente elevadas (3,26 A) y, por tanto, está sometido a fuertes desgastes.

La energía de las chispa de ignición es función de la velocidad angular del motor, decreciendo a medida que aumenta ésta.

La energía de la chispa de ignición es función de la tensión de alimentación, decreciendo a medida que decrece ésta. Este inconveniente tiene especial importancia sobre todo cuando se trata de arrancar motores fríos cuya batería tiene una carga baja. 
Los sistemas electrónicos de encendido tratan de superar, con mejor o peor fortuna, esta serie de inconvenientes $\mathrm{y}$, para ello, se han desarrollado dos sistemas fundamentales.

A través del encendido mediante transistores se trata de remediar los inconvenientes $1^{\mathrm{o}}$ y $2^{\mathrm{o}}$ del sistema convencional. Básicamente, este sistema:

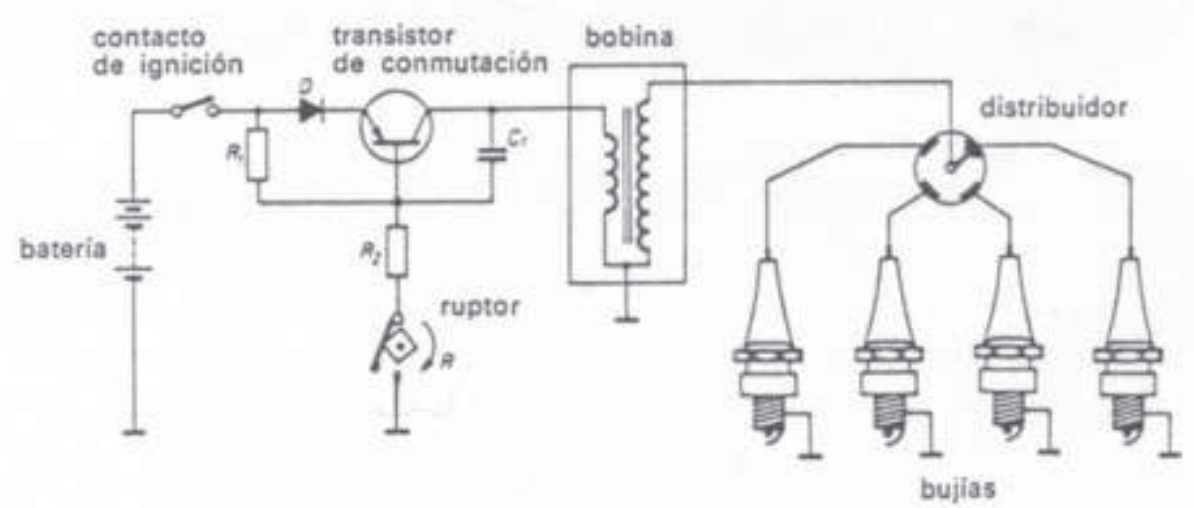

consiste en intercalar un transistor de conmutación en la alimentación de la bobina. Por el ruptor, solamente circula la corriente necesaria para excitar el transistor que, comparativamente, es mucho más pequeña que la del sistema convencional. Para solucionar los inconvenientes producidos por la inercia, vibraciones, etc. del ruptor, se adoptaron básculas electrónicas para conmutar el transistor, sincronizadas con el giro del motor por dos procedimientos fundamentales: procedimientos fotoeléctricos, y procedimientos magnéticos. 
El esquema básico del procedimiento fotoeléctrico es el representado a continuación:

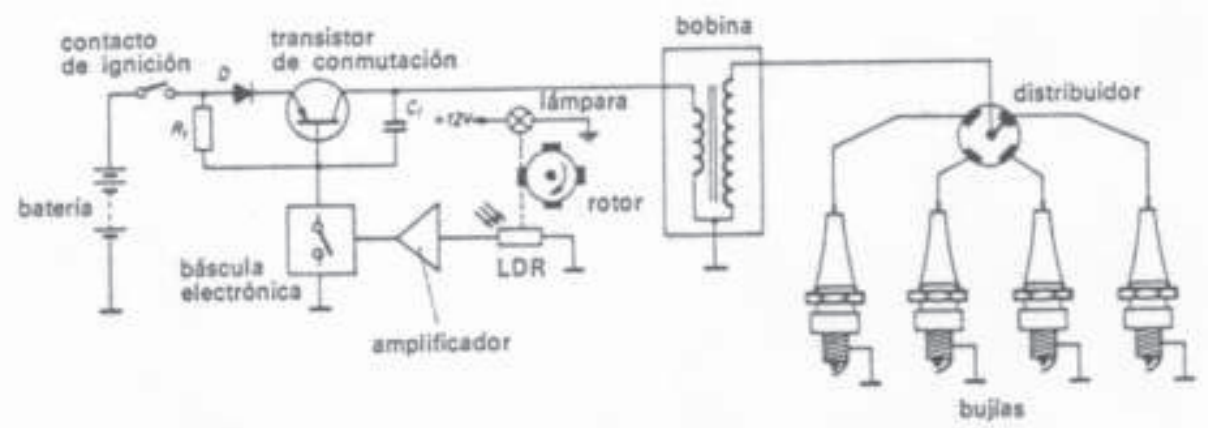

Una fotocélula, un fotodiodo o un fototransistor reciben iluminación procedente de una lamparita en determinados momentos; la conmutación provocada por estos elementos, debidamente amplificada, sincroniza la báscula electrónica que gobierna la transistor de conmutación.

El esquema básico del procedimiento magnético es el representado en el siguiente circuito:

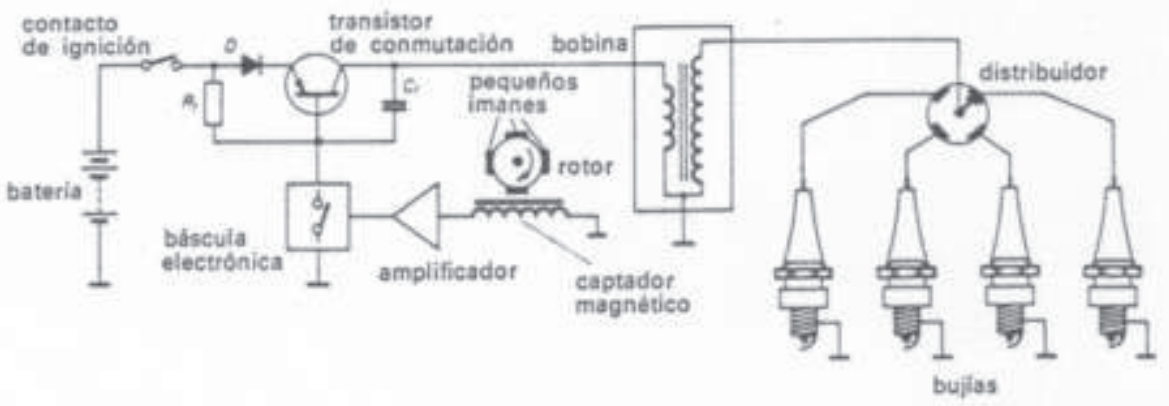

El sistema es análogo al anterior, con la única diferencia del captador que, en este caso, es magnético. 
El segundo sistema es el encendido mediante descarga de un condensador. Éste trata de remediar todos los inconvenientes del sistema convencional. Su esquema de principio es el representado seguidamente

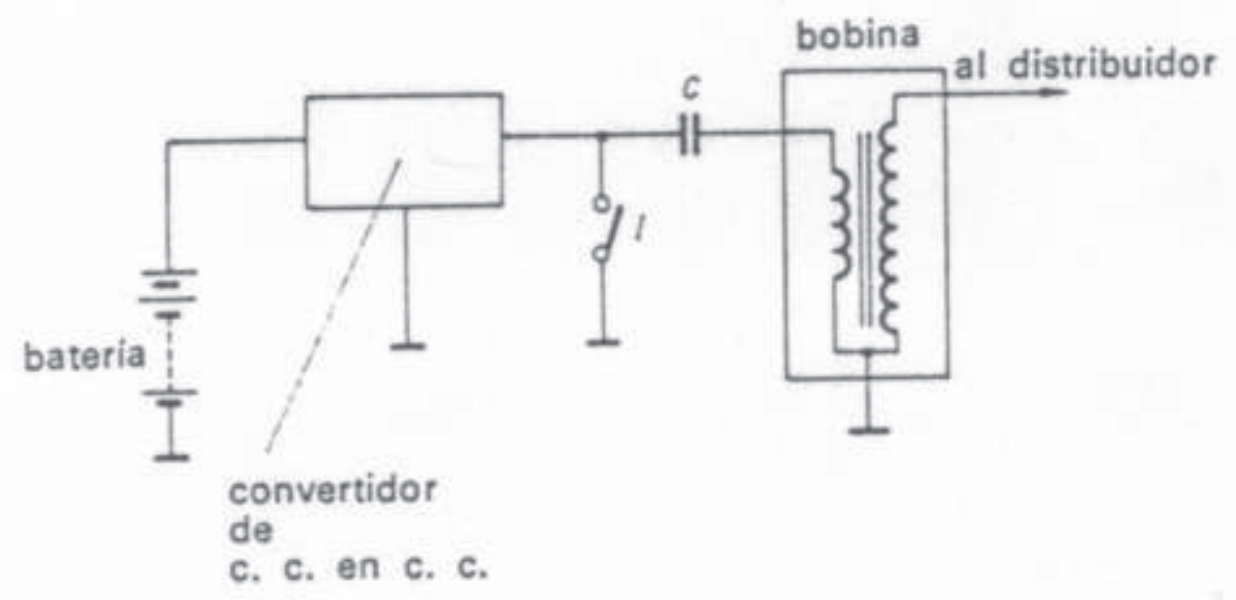

Ofrece como principal variante que la energía para la chispa no se almacena en el primario de la bobina, sino en un condensador, que se carga a partir de un convertidor de continua - continua y se descarga sobre el primario de una bobina que realmente actúa como un transformador elevador de tensión cuando se cierra el interruptor I. Para que este sistema sea realmente eficaz, deben tomarse dos precauciones fundamentales: que la chispa entregada por el circuito no sea única cuando el motor gira a bajo régimen de vueltas (para una quema de gases buena) y que el convertidor continua - continua sea estabilizado para, de esta manera, hacer independiente la tensión de la chispa de la tensión de la batería.

La tensión de salida del convertidor es fácilmente determinable: para que este sistema sea comparable al anterior, la energía acumulada en el condensador debe ser igual a la almacenada en el primario de la bobina:

$$
\begin{gathered}
W=W_{c} \\
\frac{1}{2} \cdot L_{y} \cdot I^{2}=\frac{1}{2} \cdot C \cdot V^{2}
\end{gathered}
$$


Despejando V de esta última ecuación se tiene:

$V=I \cdot \sqrt{\frac{L_{y}}{C}}$

Eligiendo un condensador de $1 \mathrm{~m} \mathrm{~F}$ :

$V=3,26 \times \sqrt{\frac{12 \times 10^{-3}}{1 \times 10^{-6}}}=3,26 \times \sqrt{12 \times 10^{3}=357,115} \approx 358$

El valor normalmente utilizado suele ser de $400 \mathrm{~V}$. Por otra parte, la impedancia de salida del convertidor debe ser baja, para permitir la carga del condensador al 95\% en un tiempo inferior al que permanece abierto el ruptor, aun cuando el motor funcione al máximo número de revoluciones; de esta manera se consigue independizar, el valor de tensión de la chispa, del número de revoluciones del motor.

Circuitos de este tipo suelen estar resueltos con configuraciones como la representada a continuación

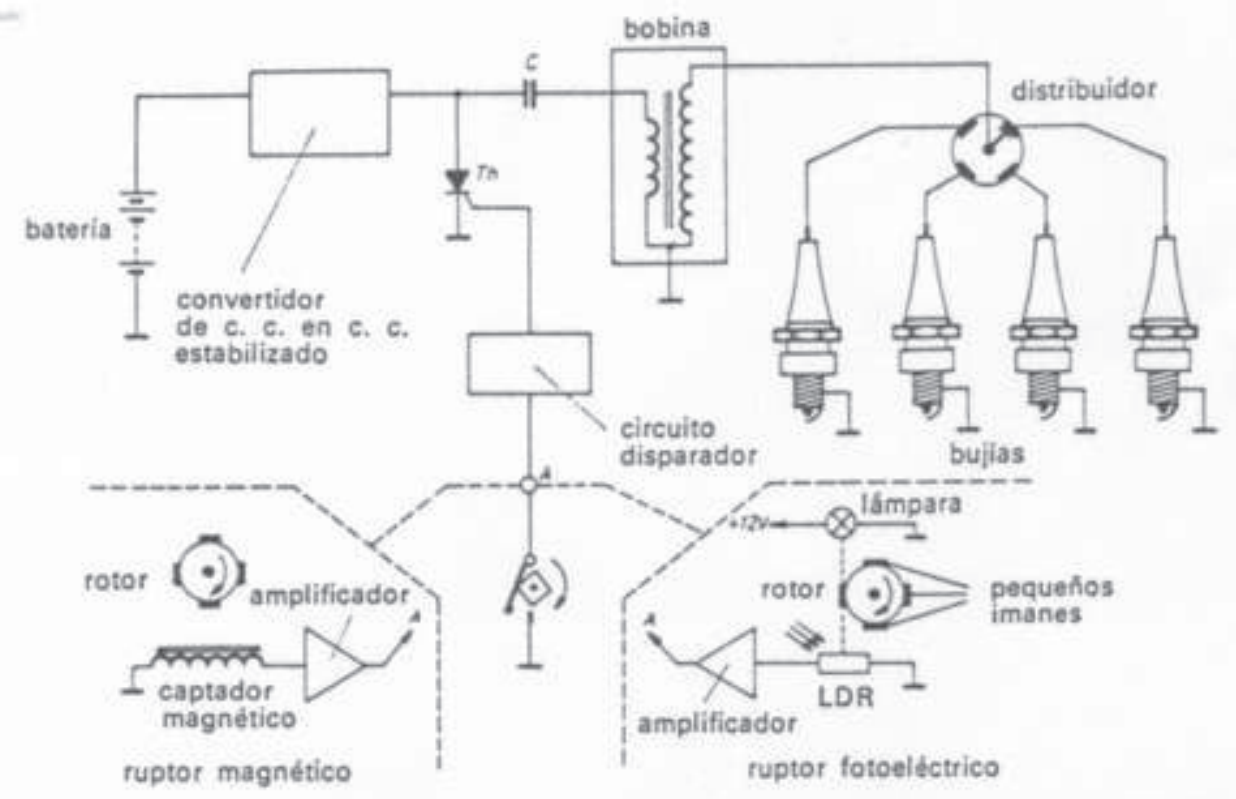


El convertidor está estabilizado y el interruptor I es sustituido por un tiristor, gobernado por un circuito disparador que, a su vez, está controlado por el ruptor. Naturalmente, en estos montajes, también el ruptor convencional puede ser sustituido por dispositivos fotoeléctricos o magnéticos.

En la figura siguiente se muestra un circuito real, desarrollado por Copresa, de encendido electrónico por descarga del condensador:

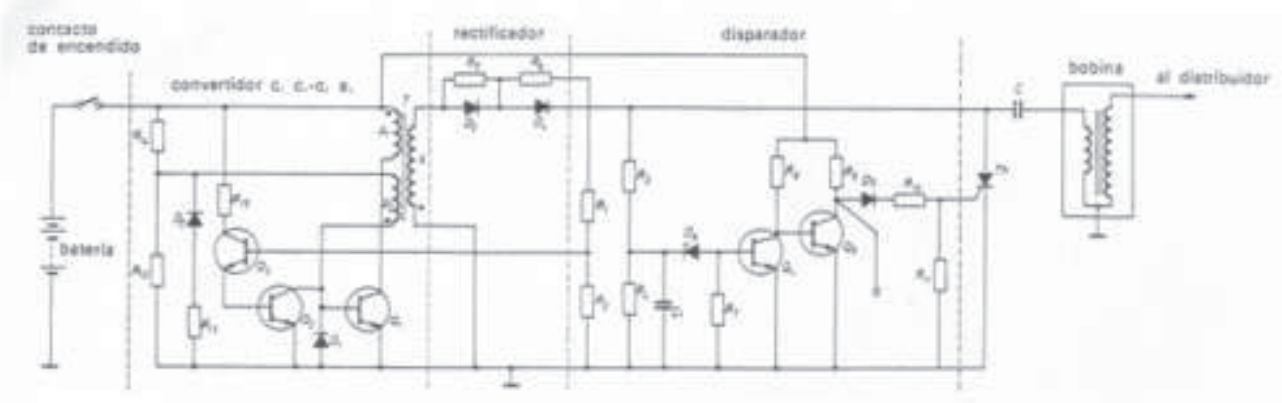

El convertidor está formado por un oscilador de bloqueo, que entrega impulsos de tensión elevada con una impedancia interna baja, impulsos que son absorbidos por el condensador, el cual paulatinamente va adquiriendo carga. Como la frecuencia del convertidor es elevada, el condensador alcanza la tensión de $400 \mathrm{~V}$ rápidamente. En ese instante, una fracción de dicha tensión producida por el divisor resistivo R1 - R2 es suficiente para enviar al convertidor la orden de paro.

Por medio del divisor R3 - R4, otra fracción de la carga de C es llevada al disparador, el cual entrega un impulso al tiristor. Este circuito disparador tiene la particularidad de que el impulso de disparo solamente alcanza al tiristor si el ruptor R está abierto. Si el ruptor permanece abierto el tiempo suficiente (régimen de giro del motor bajo), el proceso se repite varias veces proporcionando varias chispas de encendido. Por el contrario, si el ruptor está cerrado, el condensador C pierde su carga por las resistencias R1, R2, R3 y R4, hasta que entra de nuevo el convertidor que repone dicha pérdida. 
En el circuito práctico, Q1 es el transistor oscilador; y los transistores Q2 y Q3, cuando pasan a saturación, cortocircuitan la unión base - emisor de Q1, provocando el paro del oscilador. El diodo D1 protege el transistor oscilador frente a tensiones inversas. La red formada por R12, R13, R14 y D2 proporciona un arranque seguro al convertidor y evita la saturación del núcleo del transformador.

El transformador eleva la tensión de los impulsos del colector Q1, los cuales cargan el condensador C. El devanado $\mathrm{p}$ es el de realimentación del oscilador. El circuito disparador del tiristor Th está formado por los transistores Q4 y Q5 y el diodo zéner D6. Cuando la tensión en el condensador C, reducida por el divisor R3 - R4, hace que conduzca el diodo D6, el transistor Q4 pasa a saturación y el Q3 a corte. En estas condiciones, si el ruptor está cerrado, cortocircuita a masa la corriente de la resistencia de colector (R9) de Q5; pero si está abierto, esta corriente se transfiere, a través de D5 y R10, a la puerta de Th, y éste se ceba produciendo la descarga de C y una chispa en la bujía correspondiente. Obsérvese que, mediante un circuito de este tipo, se logran evitar los inconvenientes del sistema convencional del encendido:

El ruptor mecánico puede ser fácilmente sustituido por otro fotoeléctrico o magnético.

Aunque el ruptor sea mecánico la corriente que maneja es mucho menor que en encendido convencional.

La energía de la chispa no depende del régimen de vueltas de giro del motor, ya que el convertidor está diseñado para que cargue completamente al condensador C aun en los casos más desfavorables.

La energía de la chispa no depende de la tensión de la batería, pues el circuito se diseña de forma que $\mathrm{C}$ alcance $400 \mathrm{~V}$ de diferencia de potencial entre sus armaduras, aun para tensiones de batería del orden de 8 voltios.

Bueno, aquí terminamos con la electrónica básica aplicada al automóvil. Si alguien pudiera aportar sus conocimientos para expandir éste con nueva información o con 
el fin de rebatir errores que yo haya cometido en esta exposición, o manifestar críticas (a ser posible constructivas por favor) o cualquier tipo de comentario, les animo a que se dirijan a esta magna revista para que aquí me "abronquen" convenientemente por "manta" (hago aquí la solemne promesa de que, en cuanto lo sepa, ahora que manejo algo mejor, no mucho por cierto, este diabólico artefacto de los "internés de la güeb", publicaré, si ello fuere menester, claro, aquello que se me enviare).

Espero que haya resultado del gusto del lector. Si no fue así, desde aquí hago propósito de enmienda, para que el próximo artículo salga mejor (eso si no me echan antes por "rollista"). Si, por el contrario, le gustó, y despidiéndome una vez más al más puro estilo Shakesperiano, (llegará a convertirse en una tradición esta forma de despedirse y sino al tiempo) quedemos como amigos y volvamos a encontrarnos donde a la diosa fortuna más la complazca. 